\title{
Weichenstellungen für die Römischen Verträge - Akteure und Überlegungen der Bundesregierung 1955
}

\author{
Jürgen Elvert*
}

Im Jahre 2007 beging die Europäische Union den 50. Jahrestag der Unterzeichnung der Römischen Verträge. Allerdings begann der europäische Integrationsprozess, der unter anderem durch die Formulierung und Erweiterung eines Rechtskorpus von verfassungsrechtlicher Relevanz - die Konstitutionalisierung des europäischen Integrationsraumes also gekennzeichnet war, bereits fünf Jahre zuvor mit der Gründung der Europäischen Gemeinschaft für Kohle und Stahl (EGKS). Der Begriff ,Konstitutionalisierung ' taugt freilich nicht nur zur Kennzeichnung des prozessualen Charakters bei der Herausbildung eines gemeinschaftlichen Verfassungsrechts, sondern ist darüber hinaus geeignet, das Phänomen der kontinuierlichen Vertiefung der Gemeinschaftsstrukturen in seiner Gesamtheit angemessen zu beschreiben. Dabei ist aber zu berücksichtigen, dass der Begriff in den einzelnen Forschungsdisziplinen unterschiedlich bewertet wird. Einerseits attestiert man ihm ein großes Verwendungspotenzial, andererseits wird vor einem allzu inflationären Gebrauch gewarnt, weil er dann Gefahr laufe, zu einem undifferenzierten „Allerweltsbegriff“" zu degenerieren. ${ }^{1}$ Dennoch bietet ,Konstitutionalisierung ' grundsätzlich eine genügend große Offenheit, um die Entwicklung der rechtlichen Strukturen der Europäischen Union aus dem Spannungsfeld von gewollter, geschriebener und gelebter Verfasstheit heraus in ihrer ganzen Komplexität zu erfassen.

\section{Konstitutionalisierungsschübe und Phasen europäischer Integration}

Allerdings ist die damit verbundene Notwendigkeit zum abgestuften Zugang zur Frage der Verfasstheit der europäischen Gemeinschaften und ihrer Bedeutung für den europäischen Integrationsprozess mit den bisher üblichen Erklärungsmodellen aus geschichtswissenschaftlicher Sicht nicht hinreichend zu erklären. Zweifellos gibt es im Acquis communautaire Schlüsseldokumente, die als „Wegmarken“2 für den weiteren Verlauf des Konstitutionalisierungsprozesses zu verstehen sind, weil sie ihn in eine ganz bestimmte Richtung gelenkt haben. Auch gibt es bestimmte „,constitutional moments“, 3 die die Eckpunkte des Prozesses der Entstehung einer neuen Rechtsschicht ebenso wie andere zentrale konstitutionalisierungsrelevante Ereignisse markieren. Doch eignen sich solche Bezeichnungen nur bedingt zur Charakterisierung einer so komplexen Gemengelage und Abfolge von Einflüssen und Folgen, wie sie als typisch für die Konstitutionalisierung des europäischen Integrationsraums anzusehen ist. ,Wegmarke" und ,constitutional moment" suggerieren die Existenz bestimmter klar zu identifizierender Schriftsätze und Einzelereignisse, die den Verlauf des Integrationsprozesses markieren. Sie sind damit nur partiell tauglich zur Erklärung der Faktoren, die den Meinungsrahmen - die gewollte Verfasstheit also -

* Prof. Dr. Jürgen Elvert, Historisches Seminar II, Universität zu Köln.

1 Rainer Wahl: Konstitutionalisierung - Leitbegriff oder Allerweltsbegriff?, in: Carl-Eugen Eberle/Martin Ibler/ Dieter Lorenz (Hrsg.): Der Wandel des Staates vor den Herausforderungen der Gegenwart. Festschrift für Winfried Brohm zum 70. Geburtstag, München 2002, S. 191-207, hier S. 191-192.

2 Dazu: Frank R. Pfetsch (unter Mitarbeit vom Timm Beichelt): Die Europäische Union. Geschichte, Institutionen, Prozesse. Eine Einführung, 2. Auflage, München 2001, hier besonders Kapitel 1.1.

3 So Bruce Ackerman: We the people, volume 2, transformations, Cambridge/London 1998, S. 4-5. 
bestimmen, innerhalb dessen die geschriebene Verfasstheit der europäischen Gemeinschaften entstand. Sie taugen überdies ebenso wenig zur Identifikation der gelebten Verfasstheit. ${ }^{4}$

Dafür besser geeignet zu sein scheint der Begriff ,Konstitutionalisierungsschub ‘. Darunter verstehe ich einen bestimmten, aus sachlichen oder zeitlichen Gründen zusammenhängenden Abschnitt des Integrationsprozesses. Analog zu den physikalischen Gesetzmäßigkeiten bedarf der Schub stets der Konstitutionalisierungsenergie. Diese wird in einem Spannungsgeflecht aus öffentlicher Meinung, Haltung der politischen und ökonomischen Eliten oder strukturellem Reformbedarf erzeugt und kann ihre Wirkung besonders in Krisensituationen entfalten. Schubwiderstände können beispielsweise im Primat des Nationalstaatlichen liegen oder in Form von nicht steuerbaren exogenen politischen oder ökonomischen Faktoren wie politischen oder ökonomischen Krisen in Erscheinung treten beziehungsweise infolge von Fehleinschätzungen seitens der europäischen politischen Entscheidungsträger ,hausgemacht" sein. Solche Widerstände können die Konstitutionalisierung Europas verlangsamen, in eine andere Richtung lenken oder sogar stoppen. Ergänzend sei darauf hingewiesen, dass die Richtung jeden Schubes durch ,Schubumkehr' um 180 Grad geändert werden kann. Damit ist das Bild vom ,Konstitutionalisierungsschub“ frei von jedem Verdacht, so etwas wie eine Konstitutionalisierungsteleologie suggerieren $\mathrm{zu}$ wollen, was sich bei ,Wegmarke' wie , constitutional moment‘ nicht ausschließen lässt, da man sie als Markierungen eines nur in eine Richtung verlaufenden Prozesses missverstehen könnte.

Als Beispiele für Konstitutionalisierungsschübe, die den europäischen Integrationsprozess eingeleitet beziehungsweise vorangetrieben haben, seien hier dessen ,Initialzündung` zwischen 1945 und 1952 genannt, ebenso die ,Rélance Europénne“ Mitte der 1950er Jahre oder die Erweiterungen der Europäischen Gemeinschaft/Europäischen Union zwischen 1973 und 1995. Schubwiderstände gingen in diesem Zeitraum unter anderem von der Krise um die Europäische Verteidigungsgemeinschaft (EVG) und der damit verbundenen Europäischen Politischen Gemeinschaft (EPG) aus, ebenso von der sogenannten ,Krise des leeren Stuhls“ Mitte der 1960er Jahre oder auch dem Scheitern des Verfassungsvertrages infolge der ablehnenden Referenden in Frankreich und den Niederlanden im Jahre 2005. Hieran wird deutlich, dass der Integrationsprozess von den Anfängen bis zur Gegenwart von Phasen unterschiedlicher Schubdynamik gekennzeichnet ist. Wenn jedoch diese unterschiedlichen Schubphasen in Beziehung gesetzt werden zu den für den Integrationsprozess insgesamt typischen Verlaufsphasen, so wie sie sich der Geschichtswissenschaft darstellen, fällt auf, dass es nur in zwei der drei zu identifizierenden Verlaufsphasen zu einer Verringerung der Schubdynamik infolge ,Schubwiderstandes' gekommen ist.

\section{Gründungsphase}

Aus geschichtswissenschaftlicher Sicht bietet sich eine Unterteilung des Integrationsprozesses in drei Verlaufsphasen an: die Gründungsphase, die Konsolidierungsphase und die Europäisierungsphase. ${ }^{5}$ In der Gründungsphase (1952-1973) fanden sich die westeuropäischen Demokratien mit Ausnahme jener, die aus bestimmten Gründen die Exklusion vom Integrationsprozess bevorzugten, unter dem Dach supranationaler Institutionen zusammen und legten gemeinsam die Richtung des Integrationsprozesses fest. Aufgrund der erheblichen Divergenzen zwischen Supranationalisten und Intergouvernementalisten über die an-

4 Zur Geschichte des Integrationsprozesses unter besonderer Berücksichtigung externer Einflüsse auf seinen Verlauf und die damit verbundenen Interpretationsprobleme vgl. z. B.: Rudolf Hrbek/Volker Schwarz (Hrsg.): 40 Jahre Römische Verträge - Der deutsche Beitrag. Dokumentation der Konferenz anlässlich des 90. Geburtstages von Dr. h.c. Hans von der Groeben, Baden-Baden 1998.

5 Die Vorschläge zu einer Teilung des Integrationsprozesses in drei Verlaufsphasen habe ich näher erläutert in: Jürgen Elvert: Die europäische Integration, Darmstadt 2006. 
zustrebende finalité politique des integrierten Europa begann in diesem Zeitraum auch die für den heutigen Zustand der Union typische Einbeziehung föderaler und konföderaler Elemente in die Gemeinschaftsstrukturen.

\section{Konsolidierungsphase}

Die Konsolidierungsphase (1970-1992) ist einerseits markiert durch den Beitritt der drei ehemals autoritär regierten Staaten Griechenland, Portugal und Spanien, andererseits durch die systematische Vertiefung der europäischen Binnenstrukturen, so wie sie in der Einheitlichen Europäischen Akte (1987) und dem Maastrichter Vertrag (1993) zusammengefasst wurden. Sie stand unter dem Vorzeichen des auf der Haager Gipfelkonferenz im Dezember 1969 als eine Art Leitmotiv verabschiedeten Grundsatzes, dass die Erweiterung der Gemeinschaft stets mit geeigneten Maßnahmen zur Vertiefung ihrer Struktur einhergehen müsse. Damit hatten die europapolitischen Entscheidungsträger an das schon zu Beginn der Gründungsphase formulierte Ziel angeknüpft, der wirtschaftlichen die politische Integration folgen zu lassen. Entsprechende Versuche hatte es seit Gründung der EGKS wiederholt gegeben, ohne dass diese größeren Erfolg gezeitigt hätten. Erst der Präsidentschaftswechsel in Frankreich und die damit verbundene Aufgeschlossenheit der französischen Regierung für den britischen Beitrittswunsch schuf die Voraussetzung dafür, dass auf Kommissions- und Regierungsebene der Mitgliedstaaten mit Aussicht auf Erfolg über Möglichkeiten nachgedacht werden konnte, wie die Europäische Gemeinschaft den zu erwartenden Zuwachs an Neumitgliedern verkraften könnte. Somit überschneiden sich die Gründungs- und Konsolidierungsphase des Integrationsprozesses um drei Jahre. Doch während die erste Erweiterung eine Entwicklung zum Ende brachte, die bereits Ende der 1940er Jahre konzipiert und in der die ganze Bandbreite der Vorstellungen über die finalité politique des Integrationsprozesses als politische Leitbilder in den Mitgliedstaaten versammelt worden war, und die auch heute noch die einschlägige Diskussion beeinflusst, beziehen sich die in der Konsolidierungsphase angestellten Überlegungen über die Ausgestaltung der Europäischen Politischen Zusammenarbeit (EPZ) auf die Zeit nach dem Vollzug der Erweiterung und markieren damit eine neue Phase des Integrationsprozesses.

\section{Europäisierungsphase}

Die Europäisierungsphase ${ }^{6}$ (seit 1991) ist schließlich gekennzeichnet von der Überwindung der Spaltung des Kontinents durch den ,Eisernen Vorhang', womit zunächst die

6 Der Begriff ,Europäisierung “ ist, wie Kevin Featherstone gezeigt hat, vielschichtig (Kevin Featherstone: In the Name of Europe, in: Kevin Featherstone/Claudio M Radelli (Hrsg.): The Politics of Europeanization, Oxford 2003, S. 6-12). Ausgangspunkt des von mir empfohlenen Begriffsverständnisses ist die Akzeptanz der von Wolfgang Wessels vertretenen Fusionstheorie. Wessels geht davon aus, dass der Europäische Integrationsprozess von einer ,Fusion " nationaler und europäischer Politiken gekennzeichnet sei, die den traditionellen Nationalstaat zwar nicht durch eine europäische Föderation ersetzt, jedoch eine Mischung nationaler und europäischer Kompetenzen, Zuständigkeiten und Pflichten in einem Mehrebenen-System wachsender Komplexität und Undurchsichtigkeit, errichtet habe. ,Europäisierung ' wird hier als ein gradueller Prozess verstanden, der durch die Schaffung supranationaler Strukturen für die ursprünglichen sechs Mitgliedstaaten der Montanunion bzw. der Europäischen Gemeinschaften in den 1950er Jahren eingeleitet worden sei und als Folge der anschließenden Erweiterungen im Spannungsfeld einerseits nationalstaatlicher, andererseits europäischer Kompetenzen zu einer Vertiefung der Gemeinschafts- und Unionsstrukturen geführt habe, die ihrerseits weitere Anpassungen der übertragenen Kompetenzen und Verantwortlichkeiten auf supranationaler Ebene erforderlich machte, was zu einer weiteren Stabilisierung und auch Ausprägung ihrer genuin supranationalen politischen Struktur geführt habe. Dazu: Wolfgang Wessels: Zur Debatte um einen europäischen Staatenbund: Sechs Thesen der Fusionstheorie, in: Roman Herzog/Stephan Hobe: Die Europäische Union auf dem Weg zum verfassten Staatenverbund: Perspektiven der europäischen Verfassungsordnung, München 2004, S. 200 ff. Die Fusionsthese wurde erstmals vorgestellt in: Wolfgang Wessels: Staat und (westeuropäische) Integration. Die Fusionsthese, in: Politische Vierteljahresschrift, Sonderheft 23/1992 (herausgegeben von Michael Kreile), S. 36-61. 
Gründe für den Selbstausschluss einiger westeuropäischer Demokratien entfielen und die EFTA-Erweiterung von 1995 stattfinden konnte. Darüber hinaus wurden infolge des Zerfalls des Warschauer Paktes und der Sowjetunion die Voraussetzungen für die Osterweiterung der Europäischen Union geschaffen. Für die Europäische Union stellte das Ende des Kalten Krieges jedoch nicht nur eine quantitative Herausforderung dar, sondern auch eine qualitative, schließlich hatte der Ost-West-Gegensatz der späten 1940er Jahre die Einleitung des Integrationsprozesses mit vorangetrieben. Es galt also, Vorsorge- und Anpassungsmaßnahmen zu ergreifen, mit denen die Unionsstrukturen auf die Osterweiterung vorbereitet werden sollten. Und schließlich sorgten unter anderem die Implementierung des Schengener Abkommens ebenso wie die Realisierung der Wirtschafts- und Währungsunion für eine deutliche Vertiefung des Konstitutionalisierungsgrades der Europäischen Union.

Die Projektion der Konstitutionalisierungsschübe auf die Verlaufsphasen des Integrationsprozesses zeigt, dass Unterschiede in der Schubdynamik in der ersten, der Gründungsphase, zu beobachten waren, ebenso in der dritten, der Europäisierungsphase. Beide Phasen sind davon gekennzeichnet, dass es unter den beteiligten Parteien unterschiedliche Vorstellungen über den Verlauf des Integrationsprozesses gab, die Notwendigkeit einer klaren Richtungsbestimmung jedoch allgemein anerkannt war. In der Gründungsphase musste zunächst Einvernehmen zwischen Föderalisten und Konföderalisten hergestellt werden, um die Zielbestimmungen des Haager Gipfels vom Dezember 1969 zu ermöglichen, um deren Umsetzung es letztlich in der Konsolidierungsphase ging und die im Maastrichter Vertrag von 1992 realisiert wurden. Nach dem Umbruch in Osteuropa ist die Europäische Union von heute gefordert, sich neu zu erfinden und sich an die neuen internationalen Rahmenbedingungen anzupassen. Das Scheitern von zwei Referenden über den Verfassungsvertrag hatte im Jahr 2005 jedoch deutlich gezeigt, dass es innerhalb der Europäischen Union kein Einvernehmen über die Gestalt gibt, die die Europäische Union von heute auf die politischen und ökonomischen Herausforderungen von morgen vorbereiten könnte. Wie nach dem Scheitern des EVG-Projektes hat das Scheitern der zwei Referenden über den Verfassungsvertrag die Suche nach einem Ausweg aus der Krise erforderlich gemacht. Im Zentrum der nachfolgenden Überlegungen soll daher ein Blick auf die Form des Krisenmanagements geworfen werden, das auf Seiten der Bundesregierung zur Formulierung der deutschen Verhandlungsposition geführt hat, die in den Verhandlungen über die Römischen Verträge eingenommen wurde. ${ }^{7}$

\section{Entwicklung der deutschen Verhandlungsposition}

Die Rekonstruktion der Überlegungen, die im Bundeskanzleramt, Bundesaußenministerium und im Bundesministerium für Wirtschaft unter dem Eindruck der EVG-Krise und im Hinblick auf die Gestaltung der Zukunft der europäischen Integration angestellt wurden, zeigt, dass daran nur eine relativ kleine Anzahl von politischen Entscheidungsträgern beteiligt war. Zu nennen wären hier unter anderem Bundeskanzler Konrad Adenauer, Walter Hallstein als Staatssekretär im Bundesaußenministerium, Wirtschaftsminister Ludwig Erhard und Hans von der Groeben, der Generalreferent für den Schuman-Plan im Wirtschaftsministerium. Ohne damit die einschlägigen konzeptionellen Vorarbeiten und Beiträge ande-

7 Vgl. dazu auch die Beiträge von Wilfried Loth (Guy Mollet und die Entstehung der Römischen Verträge 1956/ 1957) und Hanns Jürgen Küsters (Die integrationspolitischen Wirkungen der Römischen Verträge - Eine Bilanz nach 50 Jahren) in diesem Heft. 
rer Beamter aus den Stäben der Ministerien schmälern zu wollen, ${ }^{8}$ lässt die Aktenlage den Schluss zu, dass die Position der Bundesrepublik Deutschland im Kontext der 1955/56 geführten Verhandlungen über die inhaltliche Gestaltung der Römischen Verträge im Wesentlichen von diesen vier Persönlichkeiten bestimmt worden ist. Dabei spielte Wirtschaftsminister Erhard freilich eine Sonderrolle, weil er als entschiedener Gegner einer auf einige europäische Staaten beschränkten supranational ausgerichteten ökonomischen Teil- oder Vollintegration eher als eine Art ,Agent provocateur' gesehen werden muss, dessen Kritik am europäischen Integrationskonzept in erster Linie von Hallstein und von der Groeben dazu genutzt wurde, die eigenen Argumente für eine Fortsetzung des Integrationsprozesses zu schärfen und die Bedenken des Wirtschaftsministers gegenüber dem Bundeskanzler zu entkräften.

\section{Adenauer}

Bundeskanzler Adenauer war aufgrund seiner Richtlinienkompetenz endverantwortlich für die Europapolitik der Bundesregierung. Sein Europaverständnis war zweifellos durch seine langjährige kommunalpolitische Erfahrung im Rheinland der Zwischenkriegszeit geprägt worden. Seinerzeit hatte er sich eine Verflechtung der rheinischen mit der französischen und belgischen Wirtschaft vorstellen können, womit er die politischen und ökonomischen Probleme des Rheinlandes lindern wollte. ${ }^{9}$ Nach 1945 hatte er daran angeknüpft und für eine institutionalisierte wirtschaftliche Verbindung Westdeutschlands zu Frankreich und den Benelux-Staaten plädiert. So wollte er den Zusammenhalt der Westzonen sichern und die Grundlage einer Ausgleichs- und Verständigungspolitik gen Westen schaffen, die als Mittel zum Abbau von Sicherheitsängsten seitens der Nachbarn diente sowie als Werkzeug, um eine internationale Kontrolle des Rhein-Ruhrgebiets zu verhindern. ${ }^{10}$ Nach der Gründung der Bundesrepublik Deutschland ging es ihm darum, die Westmächte davon zu überzeugen, dass die politische und wirtschaftliche Gesundung Westdeutschlands eine zentrale Voraussetzung für den Wiederaufbau des europäischen Kontinents insgesamt darstellte, die Gesundung aber einen stabilen Rahmen haben musste, den er in einer ökonomischen Verflechtung der Bundesrepublik mit den Benelux-Staaten und Frankreich, idealerweise auch noch mit Großbritannien sah. Damit wäre dann auch dem Sicherheitsbedürfnis insbesondere Frankreichs und der Benelux-Staaten gedient, zugleich schien ihm eine aktive Beteiligung des jungen westdeutschen Staates am europäischen Integrationsprojekt die beste Voraussetzung für den Abbau des Besatzungsstatuts zu sein. Dass ein solches Vorhaben die endgültige Überwindung des deutsch-französischen Gegensatzes voraussetzte, hatte Adenauer in seiner ersten Regierungserklärung am 20. September 1949 ausdrücklich betont. ${ }^{11}$

Trotz dieser Präferenz für die Integration der Bundesrepublik hatte der Bundeskanzler aber in der unter dem Eindruck der sich um 1950 abzeichnenden Verschärfung der internationalen Sicherheitslage und den in diesem Zusammenhang immer lauteren Forderungen nach

8 Zum weiteren Kreis der, Vordenker' für die bundesdeutsche Position im Rahmen der Verhandlungen über die Römischen Verträge wären noch zu nennen: Der damalige Staatssekretär im Bundeswirtschaftsministerium Ludger Westrick als Vertreter der Bundesregierung im Ministerrat der EGKS und überzeugter ,Erhardianer', ferner Franz Etzel, der Vizepräsident der Hohen Behörde, oder auch Carl Friedrich Ophüls, Leiter der deutschen Delegation bei den im Sommer 1955 begonnenen und in Messina beschlossenen Sachverständigenbesprechungen und, neben Hallstein, europapolitischer Berater des Bundeskanzlers.

9 Hanns Jürgen Küsters: Adenauers und Hallsteins Vorstellungen von Europa, in: Heinz Duchhardt (Hrsg.): Europäer des 20. Jahrhunderts. Wegbereiter und Gründer des ,modernen Europa“, Mainz 2002, S. 91-114, hier S. 92-93.

10 Ebenda, S. 85.

11 Ebenda, S. 97. 
einem westdeutschen Verteidigungsbeitrag anfangs die Aufstellung eigener westdeutscher Streitkräfte unter dem Dach der NATO bevorzugt. Erst während der Verhandlungen über den Vorschlag des französischen Ministerpräsidenten Pleven vom 24. Oktober 1950 über die Schaffung einer europäischen Armee unter einem europäischen Verteidigungsminister hatte er sich von der integrationspolitischen Bedeutung der EVG, insbesondere aber der EPG als geplantes institutionelles Dach für EVG und EGKS überzeugen lassen. Insofern ist seine verbitterte und niedergeschlagene Reaktion auf die Nachricht von der Nicht-Behandlung des EVG-Vertrages durch das französische Parlament am 30. August 1954 verständlich und für unser Thema von Bedeutung, da er bereits am nächsten Tag drei Richtlinien für die Fortführung der deutschen Politik aufstellte: ${ }^{12}$ die Fortsetzung der europäischen politischen Integration unter Zurückstellung der sicherheitspolitischen Konzepte, die Erlangung voller Souveränität für die Bundesrepublik und der Eintritt der Bundesrepublik in die NATO. Damit war der Weg abgesteckt, den die deutsche Europapolitik in den kommenden Jahren nehmen sollte.

\section{Die Hallstein-Denkschrift}

Innerhalb weniger Monate konnten die Punkte zwei und drei erledigt werden: Die Pariser Verträge vom 23. Oktober 1954 regelten die Aufnahme der Bundesrepublik Deutschland mit eigenen Streitkräften in die NATO und beendeten das Besatzungsstatut. Als Gegenleistung akzeptierte die Bundesregierung die Stationierung von Truppenkontingenten anderer NATO-Staaten auf dem eigenen Hoheitsgebiet. In Bezug auf den ersten Punkt, die Fortsetzung der europäischen politischen Integration, legte Walter Hallstein am 30. März 1955 und damit drei Tage nach Ratifikation des Pariser Vertragswerks durch das französische Parlament und drei Tage vor dem Schreiben Paul-Henri Spaaks an die Außenminister der EGKSStaaten, das üblicherweise als Auslöser der ,Relance Européenne“ gesehen wird, eine streng vertrauliche Denkschrift vor, in der er seine Vorstellungen von der Zukunft des europäischen Integrationsprozesses darlegte. ${ }^{13}$

Seine Überlegungen waren ausdrücklich als „Erwiderung auf die Gedanken des Herrn Bundeswirtschaftsministers" überschrieben. Angesichts einer Vielzahl von Überlegungen, die im gesamten EGKS-Raum über die Zukunft des europäischen Projekts angestellt wurden, hier wäre beispielsweise an einander teilweise ausschließende Überlegungen Jean Monnets, Paul-Henri Spaaks oder Johan-Willem Beyens zu erinnern, ${ }^{14}$ die weniger ökonomische Expertise, sondern vielmehr die jeweilige nationale Interessenlage spiegelten, hatte Ludwig Erhard sich Anfang März 1955 um eine Versachlichung der Debatte bemüht und das Integrationsprojekt aus seiner Sicht beurteilt. ${ }^{15}$ Seines Erachtens gab es für die Staatenwelt Europas zwei Möglichkeiten wirtschaftlicher Kooperation - zum einen die funktionale Kooperation, zum anderen die institutionelle Integration. Während nach Meinung des Bundeswirtschaftsministers bestehende internationale Wirtschaftsbeziehungen Formen funktionaler Kooperation nach sich ziehen würden, ohne dass sich daraus zwangsläufig ein politischer Zusammenschluss ergeben müsste, setze institutionelle Integration die politische

12 Dazu: Hans-Peter Schwarz: Adenauer. Der Staatsmann 1952-1967, Stuttgart 1991, S. 142.

13 Archiv für Christlich-Demokratische Politik (ACDP), Bestand I-659 (Nachlass Hans von der Groeben), 070/2, StS AA vom 30.03.1955, streng vertraulich, Titel ,Erwiderung auf die Gedanken des Herrn Bundeswirtschaftsministers zu den Problemen der Kooperation und der Integration“.

14 Vgl. dazu z. B. Hanns-Jürgen Küsters: Die Gründung der Europäischen Wirtschaftsgemeinschaft, Baden-Baden 1982, S. 38-119.

15 Das Erhard-Memorandum wird ausführlich zitiert bei: Dieter Krüger: Sicherheit durch Integration? Die wirtschaftliche und politische Zusammenarbeit Westeuropas 1947-1957/58, München 2003, S. 375-376. 
Willensentscheidung voraus. Dennoch hielt er die Hoffnung für trügerisch, dass über die institutionelle Integration funktionale Effekte freigesetzt würden, die eines Tages zu einem politischen Zusammenschluss der beteiligten Staaten führen würden. Das lasse sich, so Erhard, am Beispiel des Schuman-Plans zeigen. Hier habe von vornherein der politische jeden denkbaren wirtschaftlichen Nutzen überwogen. Würde man diesen Ansatz nun weiterverfolgen, könne die Gefahr nicht ausgeschlossen werden, dass spontane - und damit nützliche - funktionale Einrichtungen im weltweiten zwischenstaatlichen Wirtschaftssystem durch eine Vielzahl supranationaler Institutionen konterkariert würden. Auch wenn die dafür verantwortlichen politischen Institutionen dem Grundsatz marktwirtschaftlicher Freizügigkeit gefolgt seien, tendierten sie doch zum Dirigismus, weil supranationale Institutionen stets bestrebt sein müssten, die spezifischen Einflüsse nationaler Wirtschaftspolitik auf die von ihnen zu regelnden Branchen zugunsten eines einheitlichen Marktes auszuschalten. Damit würde der Nationalstaat seine wirtschaftliche Handlungsfähigkeit preisgeben, ohne dass diese von supranationalen Institutionen ersetzt werden könnte. Erhard zufolge habe institutionelle Integration daher nicht im ökonomischen, sondern im politischen Raum anzusetzen und hier sei sie mit der EVG gerade gescheitert. Vor diesem Hintergrund forderte der Wirtschaftsminister die Erprobung funktionaler Ansätze, die der politischen Unterstützung nur im Sinne der Festlegung marktwirtschaftlicher Spielregeln als Handlungsrahmen für intergouvernementale Gremien wie der Organisation für Europäische Wirtschaftliche Zusammenarbeit (OEEC), dem Allgemeinen Zoll- und Handelsabkommen (GATT), der Europäische Zahlungsunion (EZU) und dem Internationalen Währungsfonds (IWF) bedurften. ${ }^{16}$

In seiner Antwort unterstrich Walter Hallstein, dass auch Erhard die Notwendigkeit einer politischen Integration Europas anerkannt habe, ${ }^{17}$ allerdings zog Hallstein daraus völlig andere Schlüsse. Seiner Meinung nach müsse das Scheitern des EVG-Projekts als ein sowjetischer Sieg von großer Tragweite gesehen werden, da die Schaffung einer Verteidigungsgemeinschaft mit gemeinsamen Streitkräften, einem gemeinsamen Haushalt, einer gemeinsamen Rüstungspolitik die politische Integration der Gemeinschaft erheblich vorangetrieben hätte. Die NATO-Option habe zwar die sicherheitspolitische Katastrophe abwenden können, sei jedoch in Bezug auf den Integrationsprozess nicht förderlich. ${ }^{18}$ Daraus folgerte der Staatssekretär, dass die politische Integration möglichst rasch verwirklicht werden müsse. Seiner Meinung nach gab es nur ein relativ kleines Zeitfenster von zwei bis fünf Jahren, um das Integrationsvorhaben erfolgreich einleiten zu können. Danach hielt er die Gefahr einer erneuten Desintegration Europas für gegeben, da dann die öffentliche Unterstützung für die Integrationsidee insbesondere bei der europäischen Jugend sinken und nationale Interessen wieder die europäische Politik prägen würden. Das wiederum würde es der Sowjetunion erheblich erleichtern, ihren eigenen Einfluss auch westlich des ,Eisernen Vorhangs“ auszubauen. ${ }^{19}$

Hallstein zufolge gab es keinen grundsätzlichen Dissens zwischen ihm und dem Wirtschaftsminister über die Notwendigkeit der politischen Integration, sondern nur unterschiedliche Vorstellungen über die Methoden, diese herbeizuführen. In diesem Zusammenhang plädierte er dafür, nicht zu viel zu verlangen. Weder sei es notwendig, das bestgeeignete Werkzeug zu benutzen noch den theoretisch am besten denkbaren Weg herauszufinden. Angesichts des engen Zeitrahmens sei es vielmehr geboten, den unter den gegebenen Umständen relativ bestgeeigneten Weg zum Ziel zu nutzen. Folgende Wege schienen ihm ungeeig-

16 Ebenda, S. 376.

17 ACDP, Bestand I-659, 070/2, StS AA vom 30.03.1955, S. 1.

18 Ebenda, S. 3.

19 Ebenda, S. 3-6. 
net: die Einleitung des politischen Integrationsprozesses durch eine europäische Verfassung, die Nutzung des Europarates als Ausgangspunkt für eine supranationale Integration Europas und die Weiterverfolgung der von Ludwig Erhard vorgeschlagenen Methode einer , funktionalen Integration" mit sekundären institutionellen Organen bloß konsultativen Charakters. Stattdessen ging er davon aus, dass die Fortsetzung und Erweiterung des EGKS-Prozesses die einzig verbliebene Möglichkeit sei. Dabei wollte Hallstein die sektorale Integration auf die Gebiete des Verkehrswesens sowie der konventionellen und nuklearen Energiegewinnung ausdehnen. Zusätzlich strebte er eine Demokratisierung der Gemeinschaftsstrukturen durch die Einrichtung eines Europäischen Parlaments als echter Legislative an. Dabei war er sich sicher, dass seine Position mit der zu vereinbaren war, die zeitgleich in den BeneluxStaaten und Italien diskutiert wurde. Auch ging er davon aus, dass die französische Regierung in absehbarer Zeit einen vergleichbaren Standpunkt einnehmen würde. ${ }^{20}$

\section{Die von der Groeben-Denkschrift}

Hier irrte Hallstein, denn es war Jean Monnet gewesen, der mittels einer erweiterten sektoralen Integration den Stillstand des Integrationsprozesses nach dem EVG-Debakel überwinden wollte. Dagegen wurde in den Benelux-Staaten über einen neuen, horizontalen Integrationsansatz nachgedacht, der letztlich die Nationalwirtschaften der beteiligten Staaten zu einem gemeinsamen Binnenmarkt verschmelzen sollte. Dieser Gedanke war insbesondere bei Hans von der Groeben auf großes Interesse gestoßen, der nur sechs Wochen nach der Spaak-Initiative, am 13. Mai 1955 ein Aide-Memoire zur Zukunft des Integrationsprozesses präsentierte, in welchem er keinen Zweifel daran ließ, dass nur über die horizontale Gesamtintegration eine Integrationsdynamik entfaltet werden konnte, die groß genug war, um den ins Stocken geratenen Integrationsprozess wieder neu zu beleben. ${ }^{21}$

Von der Groeben konnte sich dabei auf Überlegungen stützen, die einige Monate zuvor im Wirtschaftsministerium angefertigt worden waren. ${ }^{22}$ Die Verfasser dieser 41-seitigen Studie hatten das EVG-Debakel zum Anlass genommen, um, ausgehend von den Entwicklungsmöglichkeiten der Montanunion, darüber nachzudenken, was die wirtschaftliche Integration Europas in concreto bedeutete und welche Schritte unternommen werden müssten, um eine noch näher zu definierende wirtschaftliche Integration zu verwirklichen. Auch für das Integrationsziel gab es ihrer Meinung nach unterschiedliche Zielvorgaben. So könnte es sich dabei entweder um die Intensivierung der Außenhandelsbeziehungen und ihre Stabilisierung auf hohem Niveau handeln, um die Errichtung eines neuen großen Wirtschaftsraums oder eben um die Fortsetzung der mit der Montanunion begonnenen Versuche zum politischen und wirtschaftlichen Zusammenschluss Europas. Dabei müsse geprüft werden, ob die Ziele miteinander zu vereinbaren seien, sich überschneiden oder sich gar ausschließen würden. Letztlich kam die Studie zu dem Ergebnis, dass bis zur Verwirklichung eines Systems des freien Welthandels noch viele Schwierigkeiten zu überwinden seien und es deshalb voraussichtlich zunächst nicht für alle Länder und auch insoweit nur stufenweise eingeführt werden könne. Wenngleich eine Erhöhung des Austauschvolumens im Welthandel erwartet werden könne, sei eine Steigerung der Arbeitsteilung im weltweiten Rahmen aufgrund zu geringen Interesses der au-

20 Ebenda, S. 6-10.

21 ACDP, I-659, 065/1, vom 13.05.1955.

22 ACDP, I-659, 070/2, Vertraulich, 20. Ausfertigung. Die undatierte Denkschrift enthält einen handschriftlichen Vermerk von der Groebens aus dem Jahre 1994, aus dem hervorgeht, dass sich ihre Verfasser aus seinen Unterlagen nicht rekonstruieren ließen, er jedoch davon ausging, dass es sich um eine Gemeinschaftsarbeit der Abteilungen III D und I A des Bundesministeriums für Wirtschaft gehandelt habe. Von der Groeben selbst hat die Denkschrift auf der ersten Seite mit der Jahreszahl 1954/55 versehen. 
Bereuropäischen Länder daran nicht zu erwarten, weil die regional unterschiedlich gelagerten Interessen insbesondere der außereuropäischen Länder weder die notwendigen Vereinbarungen über die vollständige Einführung des Systems zulassen würden noch erwartet werden könne, dass das System bei Schwierigkeiten in einzelnen Gebieten stabil bleibe. ${ }^{23}$

Die Denkschrift kam daher zu dem Ergebnis, dass es möglich sein könnte, in einem begrenzten Raum mit gleicher Interessenlage und vergleichbaren Wirtschaftsstrukturen Vereinbarungen zu treffen. Sofern es gelänge, in einem solchen Gebiet (also in West-/Europa) die Voraussetzungen des multilateralen Systems vollständig zu schaffen, könnte sich in einem solchen Raum eine gesteigerte Arbeitsteilung im Sinne eines Binnenmarktes entwickeln. Um aber ein binnenmarktkonformes Verhalten der europäischen Produzenten, Händler und Verbraucher zu erreichen, sei es erforderlich, eine Lösung der Währungsfrage im Sinne der Herstellung ,echter ' Wechselkurse zu erreichen, zudem müsse die Stabilität des Systems gewährleistet sein, das systemgerechte Verhalten aller Mitgliedstaaten sichergestellt und gemeinsame Maßnahmen getroffen werden für den Fall, dass die Anforderungen des Zahlungsbilanzausgleichs mit den Anforderungen der Konjunkturpolitik in Konflikt geraten sollten. Überdies müsste es zur Beseitigung von Wettbewerbsverfälschungen von Industrie zu Industrie kommen, eine Wettbewerbsordnung erstellt werden und eine fortschreitende Beseitigung der Zölle innerhalb des Wirtschaftsgebietes erfolgen. Ein Binnenmarkt sei, so fuhren die Autoren der Denkschrift fort, durch folgende Kriterien gekennzeichnet: durch Freizügigkeit der Menschen, durch zoll- und kontingentfreie Beweglichkeit der Güter und Kapitalien, durch eine einheitliche Währung, durch relativ einheitliche Verkehrstarife, durch eine einheitliche Wirtschafts- und Sozialpolitik, durch im ganzen einheitliche Rechtsnormen sowie durch einen hohen Sicherheitsgrad beim Bezug von dauerhaften Gütern und Ersatzteilen. ${ }^{24}$ Solche Merkmale würden sich beim Zusammenschluss bisher selbstständiger Wirtschaftsgebiete aber nur dann sofort erreichen lassen, wenn gleichzeitig die wirtschafts- und finanzpolitischen Zuständigkeiten zentralisiert würden, also nur in einem europäischen Bundesstaat oder vielleicht Staatenbund, mit beiden Optionen wurde aber auf absehbare Zeit nicht gerechnet.

In Bezug auf die Montanunion stellte die Denkschrift fest, dass es bislang keine Regelung der Währungsfrage gab und dass angesichts dessen ein , binnenmarktgerechtes ‘ Verhalten der Beteiligten im Montanmarkt nicht erwartet werden könne. Statt eines ,gemeinsamen“ Marktes habe die EGKS aneinandergefügte Teilmärkte mit gewissen Spielregeln geschaffen. Angesichts der ausgebliebenen Regelung der Währungsfrage sei zudem kein Subventionsverbot möglich, auch könne es nicht zu einer Harmonisierung einzelner Kostenfaktoren kommen, was wiederum die Einführung einer allgemein gültigen Wettbewerbsordnung unterbinde. ${ }^{25}$ Was die Weiterentwicklung des Montanmarktes betraf, riet die Denkschrift ausdrücklich von einer weiteren sektoralen Integration ab, da das nur zu einer Potenzierung der bisherigen Probleme führen würde, und erklärte stattdessen ,die Planung und Vorbereitung eines echten Gemeinsamen Marktes im dargestellten Sinn eine der schönsten Aufgaben der Montanunion" ${ }^{26}$ Aus wirtschaftlicher Sicht setzte das die de facto Konvertibilität mit ,echten 'Wechselkursen bei voller Liberalisierung und Abbau von Exportsubventionen und Einfuhrbelastungen voraus, ferner das Zusammenwirken der Beteiligten bei der Erhaltung, echter' und ,fester' Wechselkurse, den Abbau der Binnenzölle zum Zwecke einer Zollunion mit harmonisierten Außenzöllen, die Behandlung der Wettbewerbsverfälschungen, die Erstel-

23 Ebenda, S. 1-5.

24 Ebenda, S. 31.

25 Ebenda, S. 32-37.

26 Ebenda, S. 38-39. 
lung einer Wettbewerbsordnung sowie die Zusammenarbeit bei wichtigen wirtschaftlichen Fragen. Um diese Ziele zu erreichen, sei die Einrichtung einer übergeordneten Instanz erforderlich, dabei wurde offen gelassen, ob die unter dem Dach der EGKS entwickelten supranationalen Formen ohne Änderung übernommen werden konnten. ${ }^{27}$

Fazit

Die Akten, die im Vorfeld und während der in Messina am 1. Juni 1955 beschlossenen Regierungskonferenz auf bundesdeutscher Seite entstanden sind, zeigen, dass die auf Sachverständigenebene im Bundeswirtschaftsministerium Anfang 1955 angefertigten Überlegungen über die Notwendigkeit der horizontalen Wirtschaftsintegration, die von Hans von der Groeben in den Entscheidungsfindungsprozess eingebracht worden waren, die Position der Bundesrepublik Deutschland maßgeblich bestimmt haben. In einer Denkschrift über die ,funktionelle oder horizontale Integration“ 28 vom 6. Juli 1955 legte die Bundesregierung fest, dass auf der am 1. Oktober 1955 beginnenden Brüsseler Regierungskonferenz eine grundsätzliche Einigung zwischen den sechs beteiligten EGKS-Staaten über die Errichtung eines Gemeinsamen Marktes und die dazu erforderlichen Voraussetzungen angestrebt werden solle. Angesichts der in der Sechsergruppe divergierenden Meinungen über die Zielrichtung des Integrationsprozesses müsse dabei jeder Perfektionismus vermieden, aber Klarheit über die Grundsätze erzielt werden. Überdies sei Einigung über die Einsetzung eines ständigen Konsultativorgans zu erzielen, das unter der Verantwortung des EGKS-Ministerrates zu arbeiten hätte. Dessen Aufgaben sollten darin liegen, in Zusammenarbeit mit dem Ministerrat die Regeln für die fortschreitende Verwirklichung des Gemeinsamen Marktes zu erarbeiten, die Einzelheiten des Zollunionsvertrages vorzubereiten, dem Ministerrat Vorschläge für eine bessere Koordinierung der gemeinschaftlichen Wirtschafts-, Währungs- und Finanzpolitik vorzulegen und Vorschläge für die weitere institutionelle Ausgestaltung der Beziehungen zwischen den Mitgliedstaaten zu entwickeln. Hinsichtlich der insbesondere von französischer Seite favorisierten vertikalen Integration hielt die Denkschrift die Sektoren Energie, Nuklearenergie und Verkehrswesen für geeignet.

\section{Ausblick}

Der in dieser Denkschrift aufgestellte Zielkatalog für die Regierungskonferenz, die sich mit der Erarbeitung der Römischen Verträge zu befassen hatte, zeigt, dass die Bundesregierung weitgehend mit den Vorstellungen der Benelux-Staaten über die Zukunft des europäischen Integrationsprozesses übereinstimmte, während in französischen Regierungskreisen das Prinzip der sektoralen Integration bevorzugt wurde. Diese Position war allerdings nicht auf Grundlage des Benelux-Memorandums vom 18. Mai 1955 erarbeitet, sondern bereits unter dem Eindruck des gescheiterten EVG-Projekts entwickelt worden. Daran beteiligt war nur eine kleine Gruppe hochrangiger Politiker unter Führung des Bundeskanzlers, sowie einige wenige hochrangige Experten aus den Beamtenstäben der beteiligten Ministerien, ${ }^{29}$ allen voran Hans von der Groeben, dessen Ernennung zum EWG-Kommissar nach der Grün-

27 Ebenda, S. $39 \mathrm{ff}$.

28 ACDP, I-659, 070/2, vom 06.07.1955.

29 Hans von der Groeben berichtet in seinen Memoiren über „die Beschlüsse von Eicherscheid“, die im Landhaus Alfred Müller-Armacks in Eicherscheid unter Beteiligung der Minister von Brentano und Erhard, dem Vizepräsidenten der Hohen Behörde Etzel, Rust, Ophuels, Rudolf Regul (Direktor der Hohen Behörde) und von der Groeben selber gefasst wurden. Siehe hierzu: Hans von der Groeben: Deutschland und Europa in einem unruhigen Jahrhundert. Erlebnisse und Betrachtungen, Baden-Baden 1995, S. 273-274. Vgl. auch: Hans von der Groeben: Politische und wirtschaftliche Motive im Gleichklang - Die Vertragsverhandlungen aus Sicht eines Beteiligten, in: integration 3/1987, S. 116-120, hier S. 116. 
dung der drei Europäischen Gemeinschaften insofern nur als konsequent anzusehen ist. Diese kleine Gruppe ausgewiesener Spezialisten und erklärter ,Europäer" hatte sich vor dem Hintergrund der Herausforderung, die es zu bewältigen galt, binnen kurzer Zeit zu den ,Vordenkern' des Integrationsprozesses in bundesdeutschen Regierungskreisen entwickelt. Angesichts der aktuellen Krise des Integrationsprozesses könnte ihr damaliger Ansatz erneut einen gangbaren Weg aufzeigen: Perfektionismus vermeiden, aber Klarheit und Standfestigkeit in den Grundsätzen zeigen. Nicht zuletzt deshalb trug ihr Einsatz wesentlich dazu bei, dass die Verhandlungen im Rahmen des Messina-Prozesses trotz keineswegs günstiger äußerer Umstände zügig voranschritten.

Und so konnte Bundeskanzler Adenauer am 6. November 1956 in einer Unterredung mit dem französischen Ministerpräsidenten Guy Mollet einen weiteren Tagesordnungspunkt mit den Worten ,et maintenant, il faut faire l'Europe“30 einleiten. Dabei ging es um die deutschfranzösische Verständigung über das weitere Vorgehen in den Verhandlungen über den Gemeinsamen Markt. Zuvor war in den Luxemburger Verträgen vom 27. Oktober der Verbleib des Saarlandes bei der Bundesrepublik Deutschland beschlossen und damit ein großer Zankapfel in den deutsch-französischen Beziehungen beseitigt worden. Nun konnten die beiden Regierungen sich der Frage nach der Fortsetzung des europäischen Integrationsprozesses und damit neben der Suez-Krise der anderen großen politischen Herausforderung dieser Tage widmen. Auch wenn auf der Messina-Konferenz und in der daran anschließenden Arbeit der Expertenausschüsse bereits die groben Umrisse der künftigen europäischen Gemeinschaften entworfen worden waren, galt es doch, in einigen zentralen, aber strittigen Punkten Einvernehmen zu erzielen. Mollet und Adenauer verständigten sich daher auf die Einsetzung einer kleinen binationalen Expertenkommission unter Führung Robert Marjolins und Karl Carstens, ${ }^{31}$ die mit Erfolg nach Kompromissen in den ungeklärten Fragen suchte, welche sich primär auf die Kompetenzbereiche des geplanten Gemeinsamen Marktes im Bereich der Sozialleistungen, des Arbeitsrechtes und bestimmter Schutzklauseln für die Industrie bezogen. Damit war der Weg zur Unterzeichnung der Römischen Verträge am 25. März 1957 frei.

30 Zitiert nach: Krüger: Sicherheit, 2003, S. 440.

31 Von der Groeben: Deutschland und Europa, 1995, S. 282. 